\title{
Whose hand is this? Differential responses of right and left extrastriate body areas to visual images of self and others' hands
}

\author{
Francesco De Bellis $^{1} \cdot$ Luigi Trojano $^{1} \cdot$ Domenico Errico $^{1} \cdot$ Dario Grossi $^{1}$. \\ Massimiliano Conson ${ }^{1}$
}

Published online: 23 May 2017

(C) Psychonomic Society, Inc. 2017

\begin{abstract}
The extrastriate body area (EBA) is involved in perception of human bodies and nonfacial body parts, but its role in representing body identity is not clear. Here, we used on-line high-frequency repetitive transcranial magnetic stimulation (rTMS) to test the role of EBA in self-other distinction. In Experiments 1 and 2 we compared rTMS of right EBA with stimulation of left ventral premotor cortex (vPM), whereas in Experiment 3 we compared stimulation of right and left EBA. RTMS was applied during a hand laterality task in which self or others' hand images were presented in firstversus third-person view (Experiments 1 and 3), or while participants had to explicitly recognize their own hands (Experiment 2) presented in first- versus third-person view. Experiment 1 showed that right EBA stimulation selectively speeded judgments on others' hands, whereas no effect of left vPM stimulation was found. Experiment 2 did not reveal any effect of rTMS. Experiment 3 confirmed faster responses on others' hands while stimulating right EBA and also showed an advantage when judging self with respect to others' hands during stimulation of left EBA. These results would demonstrate that EBA responds to morphological features of human body contributing to identity processing.
\end{abstract}

Keywords RTMS · Self-other distinction · Extrastriate body area $\cdot$ Body identity $\cdot$ Visual perspective

Massimiliano Conson

massimiliano.conson@unina2.it

1 Neuropsychology Laboratory, Department of Psychology, University of Campania "Luigi Vanvitelli", Viale Ellittico 31, 81100 Caserta, Italy
A region in human lateral occipitotemporal cortex (i.e., extrastriate body area-EBA), is specifically involved in visual perception of human bodies and of nonfacial body parts (Downing, Jiang, Shuman, \& Kanwisher, 2001; Downing \& Peelen, 2015; Peelen \& Downing, 2007). Some studies suggest that this area can differently process information about oneself and other persons (Chan, Peelen, \& Downing, 2004; Myers \& Sowden, 2008; Ramsey, van Schie, \& Cross, 2011; Saxe, Jamal, \& Powell, 2006; Urgesi, Candidi, Ionta, \& Aglioti, 2007; Vocks et al., 2010), whereas others advocate the role of EBA in body perception independently of person identity (Downing \& Peelen, 2011; Hodzic, Kaas, Muckli, Stirn, \& Singer, 2009; Hodzic, Muckli, Singer, \& Stirn 2009; Peelen \& Downing, 2007). Person identity is often extracted from facial cues, but morphological features of the human body can be used to determine person identity particularly when the face is poorly visible (Downing \& Peelen, 2011; Urgesi et al., 2007).

One method to explore self-other distinction is related to manipulation of visual perspective: A human body part, such as a hand, may be observed from a first-person perspective, consistent with looking at one's own body (e.g., back of the hand with fingers pointing up), or from a third-person perspective, consistent with looking at someone else (e.g., back of the hand with fingers pointing downwards; Brady, Maguiness, \& Nì Choisdealbha, 2011; Chan et al., 2004; Nì Choisdealbha, Brady, \& Maguiness, 2011; Saxe et al., 2006). Two studies compared EBA's response to whole bodies (Chan et al., 2004) or to body parts (i.e., hands and feet; Saxe et al., 2006) presented in first- or third-person view. Results from both studies show enhanced activation of right EBA to presentation of bodies and body parts from third-person view, whereas in left EBA no viewpoint-dependent difference was observed. In their experiment, Chan et al. (2004) also tested the effect of body identity by comparing self versus familiar others' bodies 
and did not find differences in EBA activation in either hemisphere. Further studies specifically investigating EBA's response to identity, without controlling for visual perspective, provided mixed results. In particular, in two related studies, Hodzic, Muckli, et al. (2009) and Hodzic, Kaas, et al. (2009) reported conflicting findings. Contrasting self versus familiar others' full-body images (in a neutral, frontal upright posture), Hodzic, Kaas, et al. (2009) found no difference in EBA activation, whereas contrasting self versus unfamiliar others' bodies the authors found a stronger response to self than others' bodies in left EBA (but not in the right EBA). However, in another study the contrast of self versus unfamiliar others' images did not lead to differential EBA activation (Hodzic, Muckli, et al., 2009). Enhanced EBA activation in the right hemisphere has been reported during presentation of self versus others' hands in first-person perspective (Myers \& Sowden, 2008), and of self versus others' full-body images presented in several, not controlled, perspectives (Vocks et al., 2010).

Available inconsistent evidence on the role of EBA in processing body identity (Peelen \& Downing, 2007; Downing \& Peelen, 2011, 2015) might be partially explained by the fact that no studies reviewed above (but that by Chan et al., 2004) systematically manipulated the two key variables defining a body part as belonging to oneself or to another person (i.e., identity and perspective).

Several neuroimaging studies recently underlined the role of a functional network in the left hemisphere, involving EBA and ventral premotor cortex (vPM), in self-other distinction. In the well-known experiment on the rubber hand illusion, J. B. Limanowski and Blankenburg (2015) showed a functional coupling of EBA and vPM in the left hemisphere when participants self-referred the fake hand. J. Limanowski and Blankenburg (2016) replicated these neurofunctional results in a different behavioral paradigm in which participants passively viewed a virtual arm in a position corresponding (congruent condition) or not corresponding (incongruent condition) to the position of their own (unseen) arm. These findings would support the crucial role of left vPM in multisensory representation of one's own body and in the feeling of body ownership (Ehrsson, Spence, \& Passingham, 2004; Gentile, Björnsdotter, Petkova, Abdulkarim, \& Ehrsson, 2015). Activity of left vPM has also been related to faster responses when participants judge laterality of images of their own with respect to others' hands (i.e., "self-advantage"; Ferri, Frassinetti, Ardizzi, Costantini, \& Gallese, 2012). However, in a relevant TMS study, Urgesi et al. (2007) showed that EBA stimulation impaired discrimination of bodily forms, whereas stimulation of VPM impaired discrimination of bodily actions. These results suggested that EBA is involved in processing person's body identity, whereas vPM might be important for visual discrimination of actions independently from body identity.
In the present study, in two experiments we tested the role of right EBA and left VPM in self-other distinction by combining on-line high frequency repetitive transcranial magnetic stimulation (rTMS) with behavioral tasks requiring participants to judge laterality of either self or others' hands (implicit self-hand processing; Experiments 1) or to explicitly recognize their own hands (Experiment 2; Conson et al., 2015; Ferri et al., 2012; Ferri, Frassinetti, Costantini, \& Gallese, 2011). In Experiment 3, we ascertained possible functional specialization in self-other distinction of the left and right EBA by online rTMS applied while participants performed the hand laterality task with implicit identity processing. Critically, identity of body parts (self or other person's hands) was orthogonally varied with visual perspective (first or third person) in both the implicit and the explicit tasks.

\section{Experiment 1}

In the first experiment, rTMS of right EBA and left vPM was applied while participants performed an implicit body identity processing task (i.e., the laterality judgment task on self or others' hand images; Conson et al., 2015; Ferri et al., 2012; Ferri et al., 2011). To find that right EBA stimulation affects responses to identity or perspective of observed hands would demonstrate its involvement in extracting identity information from body parts (Chan et al., 2004; Jeannerod, 2004; Myers \& Sowden, 2008; Ramsey et al., 2011; Saxe et al., 2006; Urgesi et al., 2007). A modulation of performance by left vPM stimulation would be consistent with findings on the rubber hand illusion (Ehrsson et al., 2004; Gentile et al., 2015; J. Limanowski, \& Blankenburg, 2016; J. B. Limanowski, \& Blankenburg, 2015) and on the self-advantage (Ferri et al., 2012), suggesting a role of left vPM in self-body representation.

\section{Method}

\section{Participants}

Eighteen right-handed healthy male volunteers (age range: 21-30 years) participated in Experiment 1. Handedness was assessed by means of a brief structured interview. The subjects had no self-reported history of neurological diseases and were naïve to purposes and predictions of the study. We excluded subjects wearing rings or having easily recognizable marks on their hands (e.g., painted nails, tattoos, scars). The study was conducted in accordance with the ethical standards of the Declaration of Helsinki; written informed consent was obtained from all participants. The stimulation protocol was approved by the local ethics committee. 


\section{Stimuli}

The experimental stimuli consisted of gray-scale pictures of the dorsal view of right and left hands (e.g., Ferri et al., 2012; Ferri et al., 2011). The hands of each participant were photographed with a digital camera in a preliminary session 1 week before running the experiment. Hands were always photographed in the same position, in a controlled environment with constant artificial light and at a fixed distance from the camera lens $(30 \mathrm{~cm})$. The original images of the hands (one picture per hand) with fingers pointing upward $\left(0^{\circ}\right.$ orientation) were digitally rotated to obtain hand images in different orientations. Stimuli were presented one at a time on a 17-inch PC screen; they were large approximately $9.5 \mathrm{~cm}$ along the widest axis (about $8.4^{\circ}$ of visual angle at a viewing distance of $65 \mathrm{~cm}$ ). Hands images were presented in six orientations: three orientations compatible with first-person perspective $\left(0^{\circ}\right.$ with fingers pointing up, $45^{\circ}$ and $315^{\circ}$ clockwise), and three orientations compatible with third-person perspective $\left(135^{\circ}, 180^{\circ}\right.$, and $\left.225^{\circ}\right)$. Stimuli depicted participant's left or right hand in half trials (self trials), and other two males' (not involved in the experiment) right or left hands in the remaining trials (other trials; see Fig. 1). Each experimental session included 72 self trials and 72 other trials, for a total of 144 stimuli presented in random order.

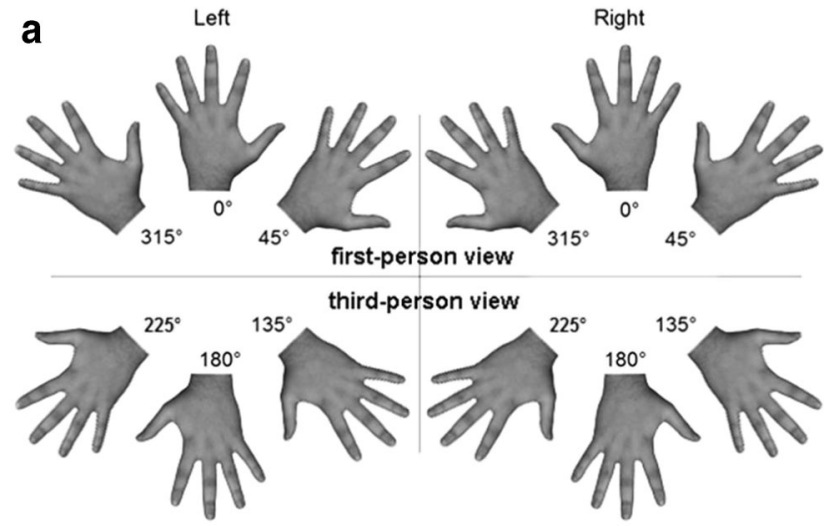

b

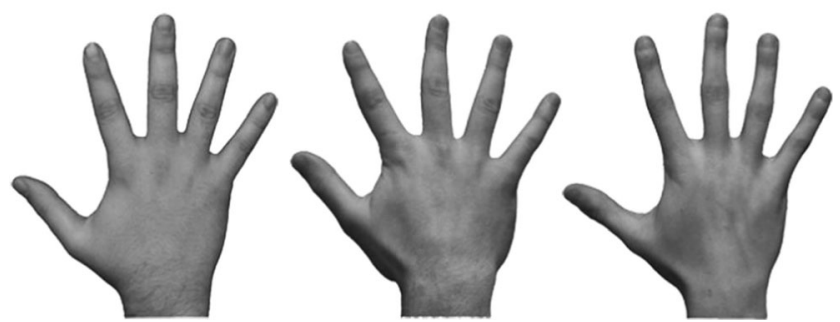

Fig. 1 Examples of the stimuli employed in the three experiments. a Left and right hands presented in six orientations (three orientations compatible with first-person perspective and three orientations compatible with third-person perspective). b Example of hands from three participants showing visual identity-related differences
Neuronavigation and TMS

On-line trains of TMS (each train including three $10-\mathrm{Hz}$ pulses, for a duration of $300 \mathrm{~ms}$ ) were delivered by means of a 70-mm figure-eight coil connected to a Magstim Rapid 2 stimulator (Magstim Company) producing a maximum output of $3.5 \mathrm{~T}$ at the coil surface (output type: biphasic; pulse width: $400 \mathrm{~ms}$ ). In keeping with safety recommendations (Rossini, Pascual-Leone, A., \& Safety of TMS Consensus Group, 2009), two consecutive stimulation trains were separated by at least 3.5 seconds, and the total number of pulses per session was 432 . Stimulation intensity (ranging from $44 \%$ to $62 \%$ of the maximum stimulator output) was set at $90 \%$ of individual motor threshold in order to reduce uncomfortable sensations possibly associated with high-intensity stimulation (Rossi et al., 2009). Participants' motor threshold was established as the lowest stimulation intensity applied over the M1 capable of evoking a visible contraction in the relaxed left hand, on at least four out of eight consecutive stimulations.

The brain targets and the scalp stimulation sites corresponding to left vPM and right EBA coordinates (Candidi, Urgesi, Ionta, \& Aglioti, 2008; Downing et al., 2001; Urgesi et al., 2007) were localized by means of Softaxic Optic (EMS) neuronavigation system. Neuronavigation was carried out on estimated-MRI stereotaxic templates of participants' brains based on a sample of 65 scalp points digitized by means of a Polaris Vicra (Northern Digital) digitizer. Mean Talairach coordinates of brain targets were as follows: right EBA: $x=50$, $y=-70, z=4$; left vPM: $x=-56, y=11, z=21$ (see Fig. 2). In the sham condition, the coil was placed at an angle of $90^{\circ}$ on the vertex, resting on the scalp with only one edge, so that the coil focus was directed away from participant head. In EBA and vPM stimulation conditions, coil positioning on the stimulation sites was checked online by means of the neuronavigator system during the entire experimental session.

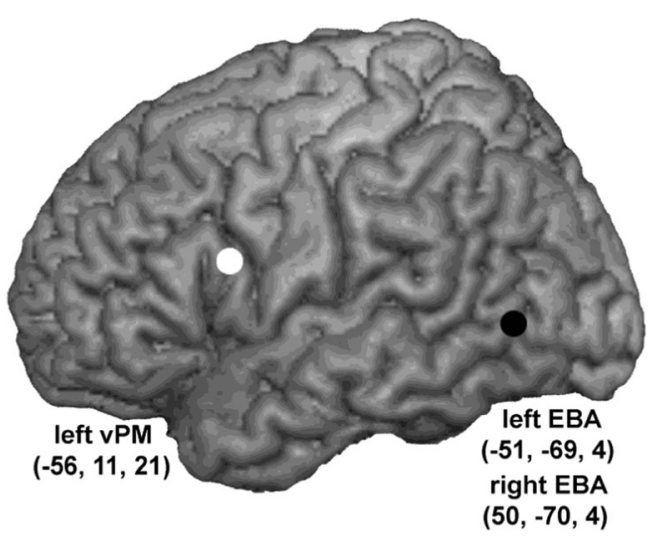

Fig. 2 Stimulation sites marked on estimated-MRI slices. Mean Talairach coordinates fell within the posterior part of the middle temporal gyrus (Brodmann's area 37) for right and left EBA and within the pars opercularis of the left inferior frontal gyrus (Brodmann's area 44) for vPM 
In all conditions, a mechanical arm fixed to a tripod held the coil.

\section{Procedures}

Participants were required to decide whether each stimulus consisted in a left or a right hand; they sat in a comfortable chair with the head on a chin rest, facing the monitor at a distance of about $65 \mathrm{~cm}$; they kept their feet resting on a foot keyboard and held their hands on their thighs, palms facing down (a cloth was laid on the subjects' hands to hide them from sight). Participants were instructed to respond as fast and accurately as possible by pressing left or right keys on a foot pedal (X-Key PS2; P.I. Engineering, Williamston, Michigan).

Each trial started with a central fixation cross, presented for $500 \mathrm{~ms}$, followed by a blank screen $(500 \mathrm{~ms})$. Afterwards, the stimulus appeared and remained visible on the screen at least $500 \mathrm{~ms}$ and until participants' response; during each stimulus presentation, one train of MRI-guided rTMS was delivered starting $150 \mathrm{~ms}$ after stimulus onset. Intertrial interval was 2,000 ms (see Fig. 3).

Our choice to deliver the TMS trains $150 \mathrm{~ms}$ after stimulus onset was based on results from a relevant study by Urgesi et al. (2007) showing that $10 \mathrm{~Hz}$ TMS trains delivered $150 \mathrm{~ms}$ after stimulus (body parts) onset affected visual discrimination of body forms when applied over EBA and affected visual discrimination of body actions when applied over vPM. In the same vein, other studies showed specific responses of the occipitotemporal cortex to presentation of bodily stimuli 100-200 ms after stimulus onset (e.g., Engell \& McCarthy, 2010; Thierry et al., 2006).

For each experiment, three experimental sessions in separate weeks were conducted, in which online rTMS was delivered over right EBA or left vPM cortex or orthogonally to the vertex (sham stimulation). The order of stimulation conditions was counterbalanced across subjects.
Accuracy and response times (RTs) were recorded for each trial. Trials with RTs shorter than $500 \mathrm{~ms}$ and outliers were discarded from analysis. Each experimental session was preceded by several practice trials without rTMS. Stimulus presentation and randomization as well as TMS triggering were controlled using Superlab 4.0 software. Data analysis was performed with SPSS V.19 software.

\section{Results and comment}

\section{Accuracy}

Percentage of correct responses (accuracy) was high in the three stimulation conditions: $92 \%$ in sham condition, $93 \%$ in the EBA condition, and $93 \%$ in the vPM condition (data for all experimental conditions are reported in Table 1). A $3 \times 2 \times 2$ repeated measures ANOVA was carried out on accuracy, with stimulation condition (right EBA, left vPM, or sham), identity (self or other) and perspective (first or third person) as withinsubjects factors. Results showed a significant main effect of perspective, $F(1,17)=16.284, p=.001, \eta_{\mathrm{p}}{ }^{2}=.489$, due to higher accuracy when participants responded to hands presented from a first-person $(98.2 \%, S E M=.53)$ than from a third-person perspective $(88.3 \%, S E M=2.63)$. No other main effect or interaction was significant (all $p \mathrm{~s}>.05$ ).

\section{$R T s$}

Trials with RTs shorter than $500 \mathrm{~ms}$ ( $2.0 \%$ of the total) and with RTs beyond the 97th or below the third percentile in each condition were discarded from analysis. A $3 \times 2 \times 2$ repeatedmeasures ANOVA was carried out on correct RTs, with stimulation condition (right EBA, left vPM, or sham), identity (self or other), and perspective (first or third person) as withinsubjects factors.

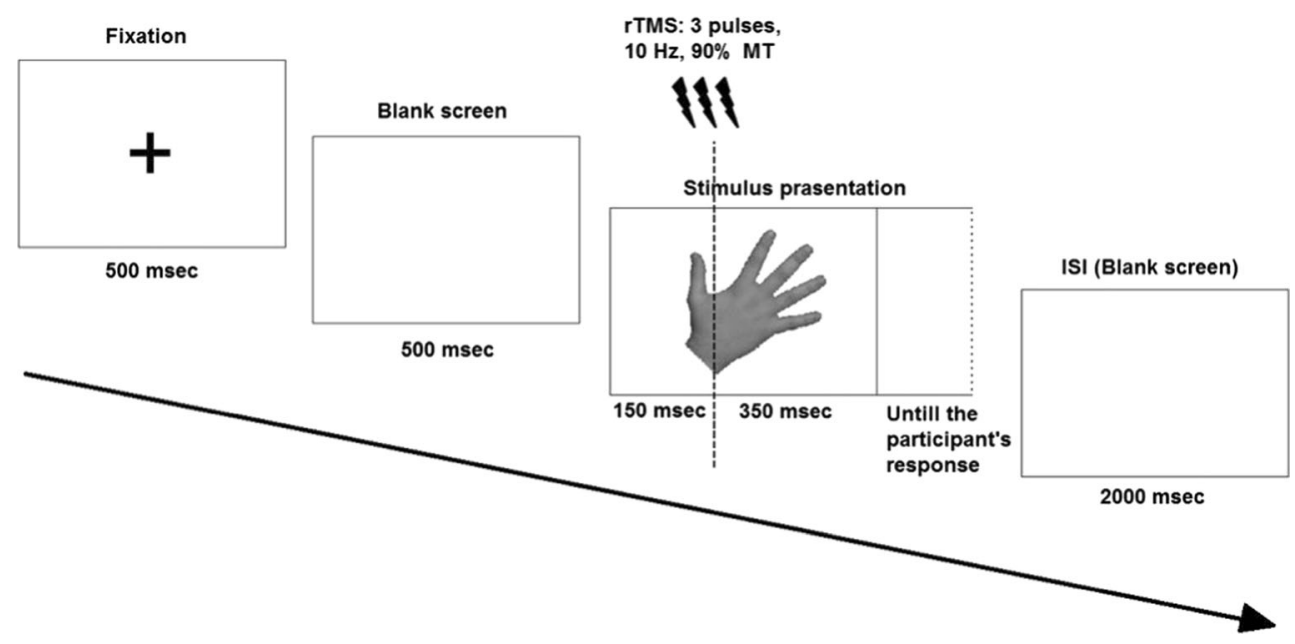

Fig. 3 Sequence of the events in the experimental trials 
Table 1 Experiment 1. Participants' accuracy (estimated mean $\pm S E M$ ) for all experimental conditions

\begin{tabular}{llllll}
\hline $\begin{array}{l}\text { Stimulation } \\
\text { conditions }\end{array}$ & \multicolumn{2}{l}{ Self hands } & & \multicolumn{2}{l}{ Other hands } \\
\cline { 2 - 3 } \cline { 6 - 6 } \cline { 5 - 6 } & First & Third & & First \\
& person & person & person & $\begin{array}{l}\text { Third } \\
\text { person }\end{array}$ \\
\hline Righ EBA & $99.4 \pm .3$ & $87.9 \pm 3$ & $99 \pm .4$ & $87.8 \pm 3.2$ \\
Left vPM & $97.3 \pm .8$ & $88 \pm 2.9$ & $98.3 \pm .6$ & $89.6 \pm 2.5$ \\
Sham & $97.8 \pm .9$ & $87.5 \pm 3.1$ & $97 \pm 1$ & $88.9 \pm 2.8$ \\
\hline
\end{tabular}

Results showed a significant main effect of perspective, $F(1,17)=313.298, p=.0001, \eta_{\mathrm{p}}{ }^{2}=.949$, due to faster responses on hands viewed in first-person (mean $=884, S E M=$ 46.1) than in third-person perspective (mean $=1,189, S E M=$ 55.1). No other main effect was significant $(p>.05)$. There was a significant interaction between stimulation condition and perspective, $F(2,34)=7.290, p=.002, \eta_{\mathrm{p}}{ }^{2}=.300$. Bonferroni-corrected post hoc comparisons showed that RTs did not differ among the three stimulation conditions (all $p$ s $>.05$ ) when participants judged first-person stimuli; instead, on third-person stimuli participants responded significantly faster in right EBA condition than in both left $\operatorname{vPM}(p=.020)$ and sham $(p=.046)$ stimulation conditions, whereas the difference between sham and left vPM stimulation was not significant $(p>.05)$.

The interaction between stimulation condition and identity was also significant, $F(2,34)=5.304, p=.010, \eta_{\mathrm{p}}{ }^{2}=$ .238. Bonferroni-corrected post hoc comparisons showed that (see Fig. 4) when judging self hands, the three stimulation conditions did not differ among each other (all $p$ s $>.05$ ); when judging others' hands, participants were significantly faster in right EBA condition than in both left $\mathrm{vPM}(p=.029)$ and sham $(p=.013)$ stimulation conditions, whereas the difference between sham and left vPM stimulation was not significant $(p>.05)$. Finally, there was a significant interaction between perspective and identity, $F(1,17)=7.546, p=.014, \eta_{\mathrm{p}}{ }^{2}=.307$. Bonferroni-corrected post hoc comparisons showed that participants' RTs were faster on self $($ mean $=871, S E M=44.2)$ than others' hands (mean $=895.4, S E M=48.2, p=.0001$ ) presented in first-person perspective, while the opposite was true for hands in third-person view, since subjects were faster on others' (mean $=1,168.3, S E M=50.82$ ) than self hands (mean $=1,210.5, S E M=60.3, p=.0001)$. No other interaction was significant $(p>.05)$.

The same $3 \times 2 \times 2$ repeated-measures ANOVA as above was also performed on participants' median RTs, computed on all correct RTs, including outliers. Consistent with the previous analysis, there was a significant main effect of perspective, $F(1,17)=114.708, p=.0001, \eta_{\mathrm{p}}{ }^{2}=.871$, due to faster RTs to hands in first-person (mean $=867$, SEM $=55.9$ ) than in third-person view (mean $=1,188.5, S E M=82.8$ ). Again, the interaction between stimulation condition and identity was significant, $F(2,34)=5.010, p=.012, \eta_{\mathrm{p}}{ }^{2}=.228$. Bonferroni-corrected post hoc comparisons revealed that when judging others' hands (see Fig. 4), participants' RTs were faster in right EBA condition than in both vPM $(p=.032)$ and sham conditions $(p=.047)$, whereas when judging self hands, no difference emerged among the three stimulation conditions (all $p \mathrm{~s}>.05$ ). Moreover, at variance with analysis on mean RTs, here we also found that RTs were significantly faster to others' than to self hands in the right EBA condition $(p=.0 .37)$.

In synthesis, the main results of Experiment 1 showed that right EBA stimulation selectively speeded judgments on others' hands, whereas no effect of left vPM stimulation was found.
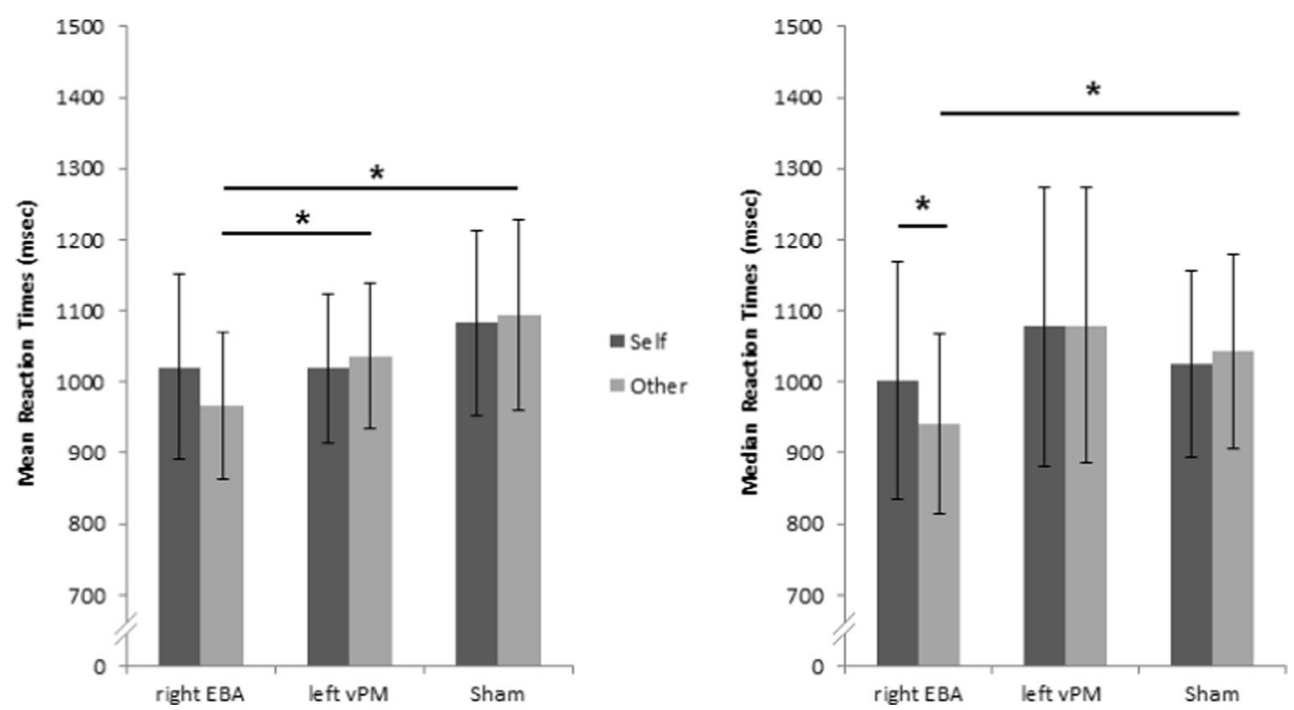

Fig. 4 Experiment 1. Mean RTs (left panel) and median RTs (right panel) - error bars are 95\% confidence intervals—showing the effect of rTMS on responses to self and others' hands (collapsing the first/third person condition). *Significant Bonferroni-corrected post hoc comparisons at $p<.05$ 


\section{Experiment 2}

Previous studies on the hand laterality task performed on self or others' hands reported a self-advantage (Conson et al., 2015; Ferri et al., 2012; Ferri et al., 2011). This selfadvantage seems to be related to activation of left vPM that, instead, is not recruited when explicitly recognizing one's own hands (Ferri et al., 2012). In Experiment 1 we did not find evidence of a self-advantage but speeded judgments on others' hands (i.e., self-disadvantage) during right EBA stimulation. Since previous behavioral experiments on explicit visual recognition of self and others' hands demonstrated a selfdisadvantage (Conson et al., 2015; Ferri et al., 2011; but see Conson, Aromino, \& Trojano, 2010), in the present experiment, rTMS of right EBA and left vPM was applied to test whether stimulation of right EBA could also enhance explicit visual recognition of others' hands.

\section{Method}

\section{Participants}

A new group of 15 right-handed healthy males (age range: 22-30 years) participated in Experiment 2. As in the previous experiment, handedness was assessed by a brief structured interview, and the subjects had no self-reported history of neurological diseases and were naïve to purposes and predictions of the study. We excluded subjects wearing rings or having easily recognizable marks on their hands (e.g., painted nails, tattoos, scars).

The study was conducted in accordance with the ethical standards of the Declaration of Helsinki; written informed consent was obtained from all participants. The stimulation protocol was approved by the local ethics committee.

\section{Stimuli, neuronavigation, and TMS}

Experimental stimuli were pictures of participants' and of other persons' hands; images were acquired and digitally manipulated as in Experiment 1 (see Fig. 1). Analogously, the brain targets and the scalp stimulation sites corresponded to left vPM and right EBA coordinates (Candidi et al., 2008; Downing et al., 2001; Urgesi et al., 2007) localized by the same methods used in Experiment 1.

\section{Procedures}

Experimental procedures were identical to those employed in Experiment 1, with the sole exception that the behavioral task here required participants to explicitly decide whether the displayed hand was or was not their own hand. The participants were required to respond as fast and accurately as possible by pressing left or right keys on a foot pedal (XKey PS2; P.I. Engineering, Williamston, Michigan).

\section{Results}

\section{Accuracy}

Percentage of correct responses (accuracy) was high in all stimulation conditions: $92 \%$ in the sham condition, $91 \%$ in the EBA condition, and $90 \%$ in the vPM condition (data for all experimental conditions are reported in Table 2). A $3 \times 2 \times$ 2 repeated-measures ANOVA was carried out on accuracy, with stimulation condition (right EBA, left vPM, or sham), identity (self or other), and perspective (first or third person) as within-subjects factors. Results did not show significant main effects or interactions (all $p \mathrm{~s}>.05$ ).

\section{$R T S$}

Trials with RTs shorter than $500 \mathrm{~ms}$ (3.1\% of the total) and with RTs beyond the 97th or below the third percentile in each condition were discarded from analysis.

A $3 \times 2 \times 2$ repeated-measures ANOVA was carried out on correct RTs, with stimulation condition (right EBA, left vPM, or sham), identity (self or other), and perspective (first or third person) as within-subjects factors. Mean RTs showing the effect of rTMS on responses to hands depending on identity (self or other) and perspective (first or third person) are reported in Fig. 5. Results did not show significant main effects or interactions (all $p \mathrm{~s}>.05$ ) with the exception of the main effect of identity that approached the significance level, $F(1,14)=$ $4.202, p=.060, \eta_{\mathrm{p}}{ }^{2}=.231$. This was due to faster responses on others' $($ mean $=981, S E M=45.4)$ than self hands (mean = 1,039, SEM $=51.3$ ).

A $3 \times 2 \times 2$ repeated-measures ANOVA as above was also performed on participants' median RTs computed on all correct RTs. No main effect or interaction was significant (all $p \mathrm{~s}>.05$ ).

In synthesis, Experiment 2 did not show any effect of rTMS on explicit hand recognition, but only revealed participants' tendency to be faster when identifying others' than self hands, in line with previous literature reporting a "self-

Table 2 Experiment 2. Participants' accuracy (estimated mean $\pm S E M$ ) for all experimental conditions

\begin{tabular}{llllll}
\hline $\begin{array}{lllll}\text { Stimulation } \\
\text { condition }\end{array}$ & \multicolumn{2}{l}{ Self hands } & & \multicolumn{2}{l}{ Other hands } \\
\cline { 2 - 3 } \cline { 6 - 7 } & $\begin{array}{l}\text { First } \\
\text { person }\end{array}$ & $\begin{array}{l}\text { Third } \\
\text { person }\end{array}$ & & $\begin{array}{l}\text { First } \\
\text { person }\end{array}$ & $\begin{array}{l}\text { Third } \\
\text { person }\end{array}$ \\
\hline Righ EBA & $92.4 \pm 4$ & $93.4 \pm 3.3$ & & $86.5 \pm 5.2$ & $84.9 \pm 4.8$ \\
Left EBA & $89.7 \pm 4.4$ & $90.2 \pm 4.2$ & $87.6 \pm 5.2$ & $85.4 \pm 5.3$ \\
Sham & $93.4 \pm 3.2$ & $94.4 \pm 3.4$ & $87.7 \pm 5.1$ & $87.8 \pm 4.7$ \\
\hline
\end{tabular}




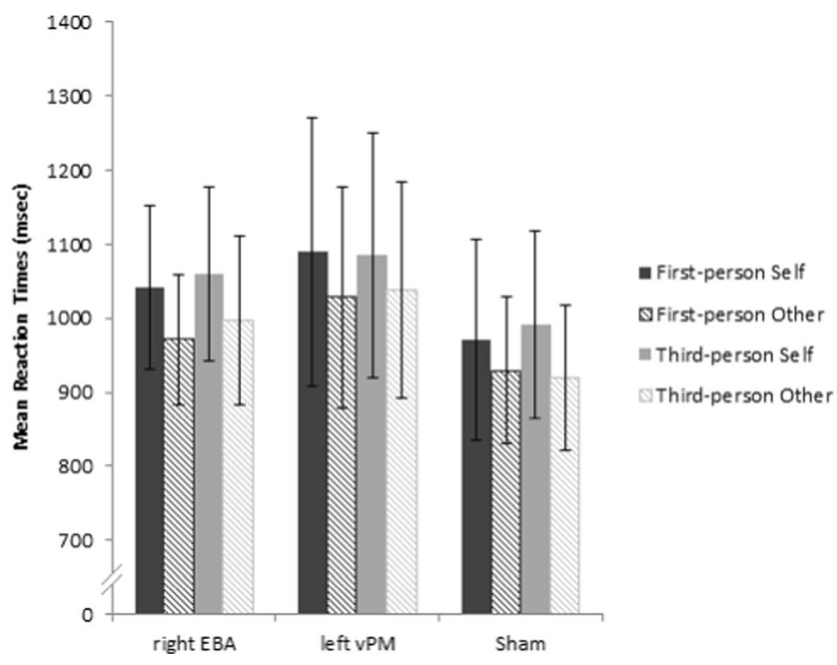

Fig. 5 Experiment 2. Mean RTs (error bars are 95\% confidence intervals) as a function of stimulation condition (right EBA, left $v P M$, or sham), identity (self or other) and perspective (first- or third-person)

disadvantage" in explicit visual recognition of self hands (Conson et al., 2015; Ferri et al., 2011).

\section{Experiment 3}

The aim of Experiment 3 was twofold. First, we wanted to replicate findings from Experiment 1, where the conservative analysis performed on median RTs showed a significant difference between right EBA and sham stimulation on RTs to others' hands, without differences related to a first- or thirdperson perspective. Therefore, in the present experiment we aimed at replicating the effect of TMS on identity discrimination, consistent with literature showing that encoding morphological features of a human body is crucial for body identity processing (Ramsey et al., 2011; Urgesi et al., 2007).

Second, we tested the role of left EBA (compared with right EBA and sham stimulation) on implicit identity processing. Recent neurofunctional studies on the rubber hand illusion showed that EBA is a node within a left-lateralized network comprising vPM and parietal cortex involved in the selfattribution of the fake body part (J. Limanowski \& Blankenburg, 2016; J. B. Limanowski \& Blankenburg 2015). In particular, Wold, Limanowski, Walter, and Blankenburg (2014) showed that during the rubber hand illusion participants misjudged their real hand's location toward the dummy hand more after left EBA than after sham rTMS stimulation. Here, we aimed at testing possible differences between left and right EBA in implicit processing of body parts identity. Following data reviewed above and consistent with results of Experiment 1, we expected that rTMS of left EBA could affect judgments to self hands, whereas stimulation of right EBA could facilitate judgments on others' hands.

\section{Method}

\section{Participants}

As stated above, one aim of Experiment 3 was to replicate the difference between right EBA and sham stimulation on RTs to others' hands. In keeping with suggestions by Simmons, Nelson, and Simonsohn $(2011,2012)$, we ran a power analysis to determine the sample size for replicating this finding. Since in the present experiment we stimulated left EBA instead of left vPM, we did not consider the effect size of the interaction between stimulation condition and identity found in Experiment 1 . Instead, we calculated the sample size as a function of the effect size of a paired $t$ test comparison between the EBA and sham conditions (Cohen $d=.77$; Morris \& DeShon, 2002), at given power level (.80) and alpha level (.05). A novel sample of 16 healthy, right-handed males (age range: 19-24 years) was thus recruited for participating in the experiment. As above, handedness was assessed by means of a brief structured interview, and the participants had no self-reported history of neurological diseases and were naive to purposes and predictions of the study. We excluded subjects wearing rings or having easily recognizable marks on their hands (e.g., painted nails, tattoos, scars). The study was conducted in accordance with the ethical standards of the Declaration of Helsinki; written informed consent was obtained from all participants. The stimulation protocol was approved by the local ethics committee.

\section{Stimuli, neuronavigation, TMS, and procedures}

The experimental stimuli were pictures of participants' and of other persons' hands; hand images were acquired and digitally manipulated as in Experiment 1 (see Fig. 1). In this case, the brain targets and the scalp stimulation sites corresponded to left and right EBA coordinates (Candidi et al., 2008; Downing et al., 2001; Urgesi et al., 2007) that were localized by means of Softaxic Optic (EMS) neuronavigation system following the same procedure described above. Mean Talairach coordinates of brain targets were as follows: right EBA: $x=50, y=-70, z=$ 4; left EBA: $x=-50, y=-70, z=4$ (see Fig. 2). Coil positioning on the stimulation sites in the three stimulation conditions (right EBA, left EBA, and sham) as well as procedures employed to run rTMS stimulation and the implicit behavioral task were the same as in Experiment 1. Participants had to respond as fast and accurately as possible by pressing left or right keys on a foot pedal (X-key XK-3; P.I. Engineering, Williamston, Michigan).

\section{Results and comment}

\section{Accuracy}

Percentage of correct responses (accuracy) was high in all stimulation conditions ( $91 \%$ in sham condition, $90 \%$ in right 
EBA condition, $93 \%$ in left EBA condition). A $3 \times 2 \times 2$ repeated-measures ANOVA was carried out on participants' accuracy, with stimulation condition (right EBA, left EBA, or sham), identity (self or other), and perspective (first or third person) as within-subjects factors. Results showed a significant main effect of perspective, $F(1,17)=21.196, p=.0001$, $\eta_{\mathrm{p}}{ }^{2}=.586$, since participants were more accurate when responded to hands in the first-person $(99.3 \%, S E M=.20)$ than in the third-person perspective $(91.641 \%, S E M=1.71)$. No other main effect or interaction was significant (all $p \mathrm{~s}>.05$ ). Table 3.

\section{$R T s$}

Trials with RTs shorter than $500 \mathrm{~ms}$ (4.1\% of the total) and with RTs beyond the 97 th or below the third percentile in each condition were discarded from analysis. A $3 \times 2 \times 2$ repeatedmeasures ANOVA was carried out on correct RTs, with stimulation condition (right EBA, left EBA, or sham), identity (self or other), and perspective (first or third person) as within-subjects factors.

Results showed a significant main effect of perspective, $F(1,15)=108.425, p=.0001, \eta_{\mathrm{p}}{ }^{2}=.878$, as the participants responded faster on hands viewed in first-person (mean = 697.96, $S E M=18.5$ ) than in third-person perspective $($ mean $=979.9, S E M=32.8)$. No other main effect was significant $(p>.05)$. As in Experiment 1, there was a significant interaction between stimulation condition and perspective, $F(2,30)=3.330, p=.049, \eta_{\mathrm{p}}{ }^{2}=.182$. Bonferroni-corrected post hoc comparisons revealed that participants' RTs did not differ among the three stimulation conditions (all $p \mathrm{~s}>.05$ ) when they judged first-person stimuli; instead, on thirdperson stimuli, participants' responses were significantly faster in the right EBA condition than in the sham $(p=.033)$ stimulation condition, whereas the right and left EBA conditions, as well as the sham and left EBA stimulations, did not significantly differ between each other (all $p \mathrm{~s}>.05$ ).

The interaction between stimulation condition and identity was also significant, $F(2,30)=5.948, p=.007, \eta_{\mathrm{p}}{ }^{2}=.284$. As in Experiment 1, Bonferroni-corrected post hoc comparisons showed that when judging self hands (see Fig. 6), the three stimulation conditions did not differ among each other $(p>$

Table 3 Experiment 3. Participants' accuracy (estimated mean $\pm S E M$ ) for all experimental conditions

\begin{tabular}{llllll}
\hline $\begin{array}{l}\text { Stimulation } \\
\text { condition }\end{array}$ & \multicolumn{2}{l}{ Self hands } & & \multicolumn{2}{l}{ Other hands } \\
\cline { 2 - 3 } \cline { 5 - 6 } & $\begin{array}{l}\text { First } \\
\text { person }\end{array}$ & $\begin{array}{l}\text { Third } \\
\text { person }\end{array}$ & & $\begin{array}{l}\text { First } \\
\text { person }\end{array}$ & $\begin{array}{l}\text { Third } \\
\text { person }\end{array}$ \\
\hline Righ EBA & $99.4 \pm .3$ & $92 \pm 2$ & $99.1 \pm .5$ & $90.5 \pm 2.3$ \\
Left vPM & $99.3 \pm .4$ & $91.7 \pm 2.2$ & & $99.4 \pm .3$ & $91.4 \pm 2.3$ \\
Sham & $99.2 \pm .4$ & $91.1 \pm 1.8$ & & $99.4 \pm .3$ & $92.9 \pm 1.6$ \\
\hline
\end{tabular}

$.05)$; instead, when judging others' hands, participants responded significantly faster in the right EBA than in the sham $(p=.012)$ stimulation condition, whereas no significant difference emerged between the sham and left EBA stimulation conditions and between right and left EBA conditions $(p>.05)$. Interestingly, Bonferroni-corrected post hoc comparisons between participants' RTs on self and others' hands did not show significant differences in the right EBA and sham stimulation conditions, whereas in the left EBA condition, a self-advantage was found, as the participants responded significantly faster to self than others' hands $(p=.010)$. No other interaction was significant $(p>.05)$.

As in the previous experiments, we also analyzed participants' median RTs computed on all correct RTs. Results of the $3 \times 2 \times 2$ repeated-measures ANOVA, with stimulation condition (right EBA, left EBA, or sham), identity (self or other), and perspective (first or third person) as within-subjects factors, showed a significant main effect of perspective, $F(1$, $15)=62.116, p=.0001, \eta_{\mathrm{p}}{ }^{2}=.805$, as RTs were faster on hands in the first-person ( mean $=657.79, S E M=18.3)$ than in the third-person perspective $($ mean $=920.18, S E M=38.89$ ). Consistent with the previous analysis, there was a significant interaction between stimulation conditions and identity, $F(2$, $30)=3.540, p=.042, \eta_{\mathrm{p}}{ }^{2}=.191$; Bonferroni-corrected post hoc comparisons showed that when judging others' hands (see Fig. 6), participants were faster in the right EBA than in the sham condition $(p=.043$,$) . Moreover, as in the analysis on$ mean RTs, a self-advantage emerged when rTMS was delivered on left EBA, since RTs were significantly faster on self than on others' hands $(p=.035)$. No other main or interaction effect was significant.

In synthesis, results of Experiment 3 confirmed the role of right EBA in processing others' hands, replicating results of Experiment 1. Importantly, we also found evidence of selfadvantage (i.e., speeded judgments on self rather than others' hands) in the left EBA stimulation condition.

\section{Discussion}

In the present study we used on-line rTMS to test the role of EBA in body identity processing. In Experiments 1 and 2, rTMS of right EBA was compared with stimulation of left vPM, whereas in Experiment 3, right and left EBA were stimulated; rTMS trains were delivered while participants judged laterality of either self or others' hands (Experiments 1 and 3) or while they had to explicitly recognize their own hands (Experiment 2).

In Experiment 1, analysis on mean RTs showed that right EBA stimulation selectively speeded judgments on hands in third-person view and on others' hands, while left vPM stimulation did not affect participants' judgments. A more conservative analysis on median RTs confirmed that right EBA 


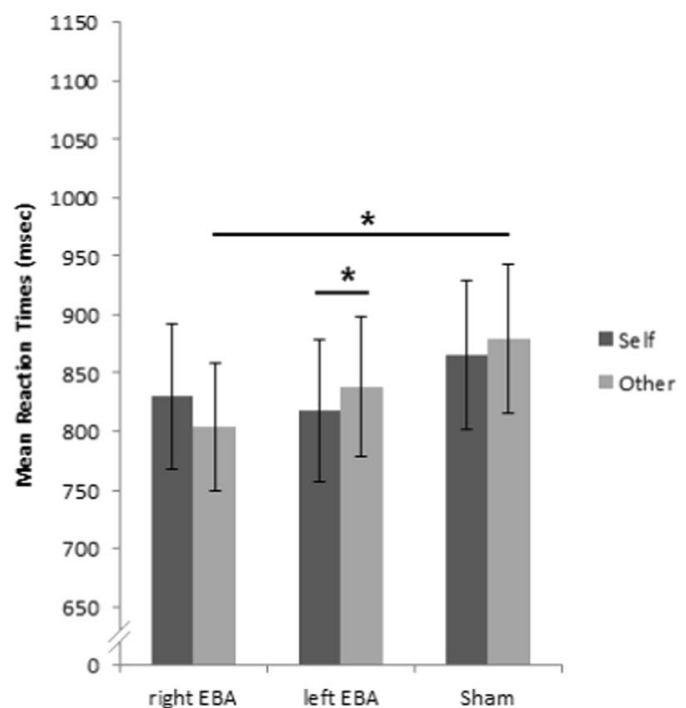

Fig. 6 Experiment 3. Mean RTs (left panel) and median RTs (right panel) - error bars are 95\% confidence intervals - showing the effect of rTMS on responses to self and others' hands (collapsing the first/

stimulation facilitated judgments on others' hands and also showed that RTs were significantly faster to others' than to self hands. Experiment 2 did not reveal any effect of rTMS on explicit recognition of self hands, whereas results of Experiment 3 supported the role of right EBA in processing others' hands as observed in Experiment 1, and suggested that left EBA is involved in processing self hands. Our discussion will be focused on data derived from the conservative analysis on median RTs only.

Some neuroimaging studies reported selectivity of right EBA activation to one's own body images (Myers \& Sowden 2008; Vocks et al. 2010), and only one study showed a specific activation of left EBA to self body (Hodzic, Kaas, et al., 2009), whereas the remaining studies on this issue found no influence of body identity on EBA activation (Chan et al., 2004; Hodzic, Kaas, et al., 2009; Hodzic, Muckli, et al., 2009). The present rTMS study clarified the role of EBA in body identity processing by demonstrating that right EBA stimulation can facilitate identification of others' hands and by suggesting that left EBA stimulation can enhance identification of self hands. Recent reviews by Downing and Peelen $(2011,2015)$ on the role of occipitotemporal cortex in body perception stated that consistent evidence supports the causal involvement of EBA in representing body shape and posture, whereas it is not clear whether EBA contributes to person identification. In particular, Downing and Peelen (2011) suggested that the role of EBA (and of fusiform body area) consists in building up a perceptual representation of the shape and posture of the body and its parts, which can be used by other brain regions to represent person identity explicitly. According to the authors, occipitotemporal cortex could process body identity only in the sense that it can distinguish between persons with different body shapes. However, other

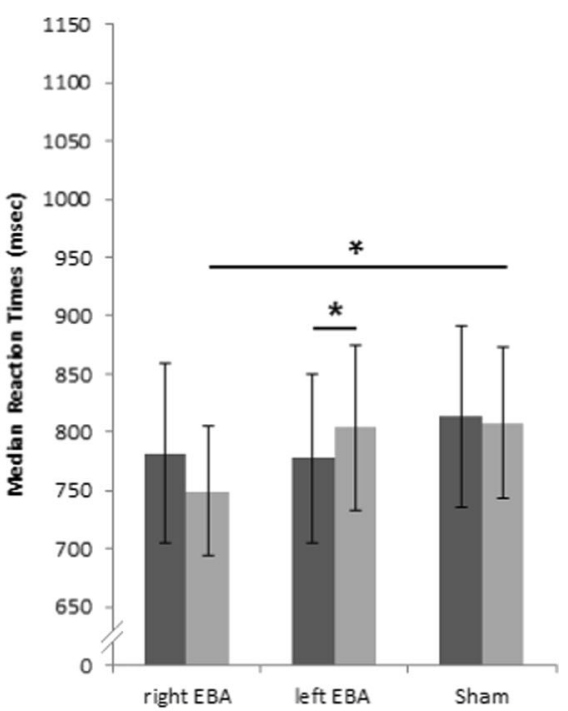

third person condition). *Significant Bonferroni-corrected post hoc comparisons at $p<.05$

authors posit that encoding body shape is a body identity processing per se, because morphological features of a human body are unique to a specific person (Ramsey et al., 2011; Urgesi et al., 2007).

These present results showing that EBA stimulation influenced body identity processing are consistent with previous results from rTMS and neuropsychological studies supporting the involvement of EBA in self-other distinction (Moro et al., 2008; Urgesi et al., 2007). Moreover, we could demonstrate that EBA stimulation affected implicit but not explicit hand recognition. Thus, if EBA is a node in the "who" system devoted to identity processing (Georgieff \& Jeannerod, 1998; Jeannerod, 2004), this region might be causally involved in implicit body identity processing, while explicit identification of who somebody is could require that other cortical nodes of the system process body identity information. This interpretation would reconcile our own and other researchers' results supporting the role of EBA in representing body identity with Downing and Peelen's proposal (2011) according to which EBA (and the fusiform body area) is part of a broader system for representing body identity: EBA would be involved in processing morphological features peculiar of a person's body that are then processed by anterior brain regions to explicitly define who a person is.

Another relevant point was the lack of effects of left vPM stimulation on self-other distinction. This finding is consistent with Urgesi et al.'s (2007) results showing that EBA is crucial for processing persons' body identity, whereas VPM is mainly involved in visual discrimination of actions without taking into account a persons' identity. However, in a recent fMRI study, Ferri et al. (2011) required participants to judge laterality of either self or others' hands and found that processing one's own hands relied upon activation of both EBA and 
premotor cortex. A previous study by Astafiev et al. (2004) demonstrated that EBA, particularly in the left hemisphere, together with sensorimotor areas, responded to self-produced body movements, even when the moving body part was not visible. Consistently, it has been suggested that left EBA would represent body parts and tools as well as related actions (Bracci, Cavina-Pratesi, Ietswaart, Caramazza, \& Peelen, 2012; Perini, Caramazza, \& Peelen, 2014). These body- and action-related representations would be used during goaldirected actions as demonstrated by TMS-related inhibition of left EBA on goal-oriented motor behavior (Zimmermann, Verhagen, de Lange, \& Toni, 2016).

Consistent with the above findings, recent neuroimaging studies on the rubber hand illusion demonstrated the crucial role of a functional network in the left hemisphere, involving EBA and vPM, in self-attribution of the fake hand. More relevant, in an rTMS study, Wold et al. (2014) showed that during the rubber hand illusion participants misjudged their real hand's location toward the dummy hand more often after the left EBA than after the sham rTMS stimulation. On this basis, one could speculate that within this functional network the left EBA plays a primary role in identifying one's own body.

As a final remark, we demonstrated that rTMS over EBA enhanced participants' performance. It is often assumed that TMS induces neuronal noise in the underlying brain tissue leading to an impairment of the respective cognitive functions. However, several studies reported a TMS-related enhancement of behavioral performance (decreased RTs or increased accuracy), suggesting that TMS can also facilitate cognitive processing (for a review, see Luber \& Lisanby, 2014). More precisely, Luber and Lisanby (2014) identified two basic categories of enhancement mechanisms: direct modulation of the cortical region expected to be specifically involved in a given task and addition by subtraction, which is a disruption of processing competing for task performance. According to the authors, a local cortical facilitation could be induced when high-frequency TMS $(10 \mathrm{~Hz})$ is administered immediately before performance, or even (at subthreshold intensities) during cortical processing. In the latter case, it has been suggested that the neural mechanism enhancing performance could be the so-called stochastic resonance effect (Luber \& Lisanby, 2014; Miniussi, Ruzzoli, \& Walsh, 2010; Schwarzkopf, Silvanto, \& Rees, 2011), according to which ongoing stimulus processing could be favored by adding low noise to neural activity through a weak TMS stimulation (i.e., stimulation under motor or visual threshold). This interpretative framework of TMS effects might explain why we found facilitatory effects, although our TMS paradigm was similar to those employed in previous studies showing an interference effect. In particular, Urgesi et al. (2007) demonstrated inhibitory effects of TMS over EBA and vPM stimulation on body form and action, respectively, by means of a protocol with a two- pulse, high-frequency $(10 \mathrm{~Hz})$ and high-intensity $(120 \%$ of individuals' MT) rTMS stimulation applied on the target areas immediately after presentation of body stimuli, which remained on view for $150 \mathrm{~ms}$. In the present experiments, we also used high-frequency $(10 \mathrm{~Hz})$ rTMS on target cortical areas, but we applied three-pulse, underthreshold trains $(90 \%$ of individuals' MT) $150 \mathrm{~ms}$ after presentation of hand images that remained on view until participants' responses. Following Luber and Lisanby's (2014) account, high-frequency (10 Hz) rTMS with trains applied immediately after $(150 \mathrm{~ms})$ stimulus presentation and while visual processing of stimulus was still occurring could account for the facilitation effect we found here. To date, explanations of precise neural mechanisms underlying TMS effects are still somewhat speculative (Luber \& Lisanby, 2014), and we cannot entirely rule out that an addition-by-subtraction mechanisms played a role in our experiments. Nevertheless, taking into account the features of our TMS protocol and the current knowledge about the functional role of both right and left EBAs, we suggested a more parsimonious interpretation of our TMS effects in terms of facilitation of cortical processing.

One limitation of the present study was that in all experiments we presented participants with their own left or right hand in half trials (self-trials), and with other two males' (not involved in the experiment) right or left hands in the remaining trials (other trials). This procedure determined an unbalanced ratio in the proportion of others' and self hands, which in its turn might have provided cues about hand identity. Related to this, we could not fully rule out that differences in visual features between self and others' hands might have played a role in the behavioral performance as well. Although our experimental set-up has been widely employed in literature (see, for instance, Conson et al., 2010; Ferri et al., 2011; Frassinetti, Ferri, Maini, Benassi, \& Gallese, 2011), future studies could control for possible confounds related to visual features of hand images and to frequency of presentation of self hands (e.g., by using images of only one participant's hands as the control images for another participant), keeping a balanced ratio between frequency of self and others' hands (see, for instance, Experiment 3 by Ferri et al., 2011).

Moreover, we did not implement a functional localizer task to identify brain targets (particularly right and left EBAs) prior to performing TMS experiments. However, it is worth noting that we used the same Talairach coordinates as in previous studies that did not employ functional localization procedures (e.g., Candidi et al., 2008; Urgesi et al., 2007). Moreover, we only focused on two brain areas, EBA and vPM, thought to be involved in self-other distinction. It is important to acknowledge that other brain areas may play a role in this cognitive capacity, such as the temporoparietal junction (e.g., Blanke, Ortigue, Landis, \& Seeck, 2002; Blanke et al., 2005; Heinisch, Krüger, \& Brüne, 2012; for a review, see Donaldson, Rinehart, \& Enticott, 2015) and that further studies are needed 
to comprehend the functional interplay between EBA and the other areas involved in self-other distinction.

In conclusion, our results strongly suggested that right EBA stimulation enhanced implicit visual processing of others' hands while stimulation of left EBA led to a self-advantage. We could thus support the key role of EBA in person identification and self-other distinction (Jeannerod, 2004; Ramsey et al., 2011). However, it remains to be elucidated the way in which EBA interact with others nodes of the "who" system in order to explicitly represent person identity.

Acknowledgements This article was supported in part by a PRIN Grant 2010 to D.G. No additional external funding was received for this study. We want to thank Francesca Garbarini for her insightful comments on earlier versions of the manuscript.

\section{References}

Astafiev, S. V., Stanley, C. M., Shulman, G. L., \& Corbetta, M. (2004). Extrastriate body area in human occipital cortex responds to the performance of motor actions. Nature Neuroscience, 7, 542-548.

Blanke, O., Mohr, C., Michel, C. M., Pascual-Leone, A., Brugger, P., Seeck, M., ... \& Thut, G. (2005). Linking out-of-body experience and self processing to mental own-body imagery at the temporoparietal junction. Journal of Neuroscience, 25, 550-557.

Blanke, O., Ortigue, S., Landis, T., \& Seeck, M. (2002). Stimulating illusory own-body perceptions. Nature, 419, 269-270.

Bracci, S., Cavina-Pratesi, C., Ietswaart, M., Caramazza, A., \& Peelen, M. V. (2012). Closely overlapping responses to tools and hands in left lateral occipitotemporal cortex. Journal of Neurophysiology, 107, 1443-1456.

Brady, N., Maguinness, C., \& Ní Choisdealbha, A. (2011). My hand or yours? Markedly different sensitivity to egocentric and allocentric views in the hand laterality task. PLOS ONE, 6, e23316.

Candidi, M., Urgesi, C., Ionta, S., \& Aglioti, S. M. (2008). Virtual lesion of ventral premotor cortex impairs visual perception of biomechanically possible but not impossible actions. Social Neuroscience, 3, 388-400.

Chan, A. W., Peelen, M. V., \& Downing, P. E. (2004). The effect of viewpoint on body representation in the extrastriate body area. Neuroreport, 15, 2407-2410.

Conson, M., Aromino, A. R., \& Trojano, L. (2010). Whose hand is this? Handedness and visual perspective modulate self/other discrimination. Experimental Brain Research, 206, 449-453.

Conson, M., Errico, D., Mazzarella, E., De Bellis, F., Grossi, D., \& Trojano, L. (2015). Impact of body posture on laterality judgement and explicit recognition tasks performed on self and others' hands. Experimental Brain Research, 233, 1331-1338.

Donaldson, P. H., Rinehart, N. J., \& Enticott, P. G. (2015). Noninvasive stimulation of the temporoparietal junction: A systematic review. Neuroscience \& Biobehavioral Reviews, 55, 547-572.

Downing, P. E., Jiang, Y., Shuman, M., \& Kanwisher, N. (2001). A cortical area selective for visual processing for the human body. Science, 293, 2470-2473.

Downing, P. E., \& Peelen, M. V. (2011). The role of occipitotemporal body-selective regions in person perception. Cognitive Neuroscience, 2, 186-203.

Downing, P. E., \& Peelen, M. V. (2015). Body selectivity in occipitotemporal cortex: Causal evidence. Neuropsychologia, 83, $138-148$.
Ehrsson, H. H., Spence, C., \& Passingham, R. E. (2004). That's my hand! Activity in premotor cortex reflects feeling of ownership of a limb. Science, 305, 875-877.

Engell, A. D., \& McCarthy, G. (2010). Selective attention modulates face-specific induced gamma oscillations recorded from ventral occipitotemporal cortex. Journal of Neuroscience, 30, 8780-8786.

Ferri, F., Frassinetti, F., Ardizzi, M., Costantini, M., \& Gallese, V. (2012). A sensorimotor network for the bodily self. Journal of Cognitive Neuroscience, 24, 1584-1595.

Ferri, F., Frassinetti, F., Costantini, M., \& Gallese, V. (2011). Motor simulation and the bodily self. PLOS ONE, 6, e17927.

Frassinetti, F., Ferri, F., Maini, M., Benassi, M. G., \& Gallese, V. (2011). Bodily self: An implicit knowledge of what is explicitly unknown. Experimental Brain Research, 212, 153-160.

Gentile, G., Björnsdotter, M., Petkova, V. I., Abdulkarim, Z., \& Ehrsson, H. H. (2015). Patterns of neural activity in the human ventral premotor cortex reflect a whole-body multisensory percept. NeuroImage, 109, 328-340.

Georgieff, N., \& Jeannerod, M. (1998). Beyond consciousness of external reality: A "who" system for consciousness of action and self-consciousness. Consciousness and Cognition, 7, 465-477.

Heinisch, C., Krüger, M. C., \& Brüne, M. (2012). Repetitive transcranial magnetic stimulation over the temporoparietal junction influences distinction of self from famous but not unfamiliar others. Behavioral Neuroscience, 126, 792-796.

Hodzic, A., Kaas, A., Muckli, L., Stirn, A., \& Singer, W. (2009). Distinct cortical networks for the detection and identification of human body. NeuroImage, 45, 1264-1271.

Hodzic, A., Muckli, L., Singer, W., \& Stirn, A. (2009). Cortical responses to self and others. Human Brain Mapping, 30, 951-962.

Jeannerod M. (2004). Visual and action cues contribute to the self-other distinction. Nature Neuroscience, 7, 422-423.

Limanowski, J. B., \& Blankenburg, F. (2015). Network activity underlying the illusory self-attribution of a dummy arm. Human Brain Mapping, 36, 2284-2304.

Limanowski, J., \& Blankenburg, F. (2016). Integration of visual and proprioceptive limb position information in human posterior parietal, premotor, and extrastriate cortex. Journal of Neuroscience, 36, 2582-2589.

Luber, B., \& Lisanby, S. H. (2014). Enhancement of human cognitive performance using transcranial magnetic stimulation (TMS). NeuroImage, 85(Pt. 3), 961-970.

Miniussi, C., Ruzzoli, M., \& Walsh, V. (2010). The mechanism of transcranial magnetic stimulation in cognition. Cortex, 46, 128-130.

Moro, V., Urgesi, C., Pernigo, S., Lanteri, P., Pazzaglia, M., \& Aglioti, S. M. (2008). The neural basis of body form and body action agnosia. Neuron, 60, 235-246.

Morris, S. B., \& DeShon, R. P. (2002). Combining effect size estimates in meta-analysis with repeated measures and independent-groups designs. Psychological Methods, 7, 105-125.

Myers, A., \& Sowden, P. T. (2008). Your hand or mine? The extrastriate body area. NeuroImage, 42, 1669-1677.

Nì Choisdealbha, A., Brady, N., \& Maguiness, C. (2011). Differing roles for the dominant and non-dominant hands in the hand laterality task. Experimental Brain Research, 211, 73-85.

Peelen, M. V., \& Downing, P. E. (2007). The neural basis of visual body perception. Nature Reviews Neuroscience, 8, 636-648.

Perini, F., Caramazza, A., \& Peelen, M. V. (2014). Left occipitotemporal cortex contributes to the discrimination of tool-associated hand actions: fMRI and TMS evidence. Frontiers in Human Neuroscience, 8, 591.

Ramsey, R., van Schie, H. T., \& Cross, E. S. (2011). No two are the same: Body shape is part of identifying others. Cognitive Neuroscience, 2, 207-208.

Rossi, S., Hallett, M., Rossini, P. M., Pascual-Leone, A., \& Safety of TMS Consensus Group. (2009). Safety, ethical considerations, and application guidelines for the use of transcranial magnetic stimulation in clinical practice and research. Clinical Neurophysiology, 120, 2008-2039. 
Saxe, R., Jamal, N., \& Powell, L. (2006). My body or yours? The effect of visual perspective on cortical body representations. Cerebral Cortex, 16, 178-182.

Schwarzkopf, D. S., Silvanto, J., \& Rees, G. (2011). Stochastic resonance effects reveal the neural mechanisms of transcranial magnetic stimulation. Journal of Neuroscience, 31, 3143-3147.

Simmons, J. P., Nelson, L. D., \& Simonsohn, U. (2011). False-positive psychology undisclosed flexibility in data collection and analysis allows presenting anything as significant. Psychological Science, 22, 1359-1366.

Simmons, J. P., Nelson, L. D., \& Simonsohn, U. (2012). A 21 word solution. Retrieved from https://papers.ssrn.com/sol3/papers.cfm? abstract id $=2160588$

Thierry, G., Pegna, A. J., Dodds, C., Roberts, M., Basan, S., \& Downing, P. (2006). An event-related potential component sensitive to images of the human body. NeuroImage, 32, 871-879.
Urgesi, C., Candidi, M., Ionta, S., \& Aglioti, S. M. (2007). Representation of body identity and body actions in extrastriate body area and ventral premotor cortex. Nature Neuroscience, 10, 30-31.

Vocks, S., Busch, M., Grönemeyer, D., Schulte, D., Herpertz, S., \& Suchan, B. (2010). Differential neuronal responses to the self and others in the extrastriate body area and the fusiform body area. Cognitive, Affective \& Behavioral Neuroscience, 10, $422-429$.

Wold, A., Limanowski, J., Walter, H., \& Blankenburg, F. (2014). Proprioceptive drift in the rubber hand illusion is intensified following $1 \mathrm{~Hz}$ TMS of the left EBA. Frontiers in Human Neuroscience, 8 , 390.

Zimmermann, M., Verhagen, L., de Lange, F. P., \& Toni, I. (2016). The extrastriate body area computes desired goal states during action planning. eNeuro, 3(2). doi:10.1523/ ENEURO.0020-16.2016 